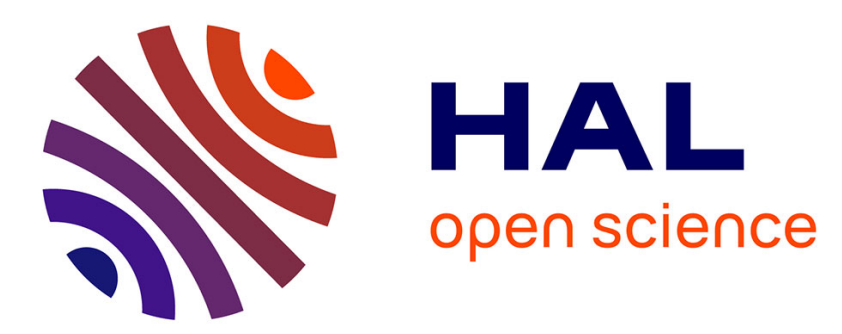

\title{
Calibration of constitutive models of steel beams subject to local buckling by using Digital Image Correlation
}

François Hild, Stéphane Roux, Néstor Guerrero, Maria Eugenia Marante, Julio Florez-Lopez

\section{- To cite this version:}

François Hild, Stéphane Roux, Néstor Guerrero, Maria Eugenia Marante, Julio Florez-Lopez. Calibration of constitutive models of steel beams subject to local buckling by using Digital Image Correlation. European Journal of Mechanics - A/Solids, 2011, 30 (1), pp.1-10. 10.1016/j.euromechsol.2010.09.007 . hal-00521187

\section{HAL Id: hal-00521187 \\ https://hal.science/hal-00521187}

Submitted on 14 Nov 2010

HAL is a multi-disciplinary open access archive for the deposit and dissemination of scientific research documents, whether they are published or not. The documents may come from teaching and research institutions in France or abroad, or from public or private research centers.
L'archive ouverte pluridisciplinaire HAL, est destinée au dépôt et à la diffusion de documents scientifiques de niveau recherche, publiés ou non, émanant des établissements d'enseignement et de recherche français ou étrangers, des laboratoires publics ou privés. 


\title{
Calibration of constitutive models of steel
}

\section{beams subject to local buckling by using}

\section{Digital Image Correlation}

\author{
François Hild $^{\mathrm{a}, *}$, Stéphane Roux ${ }^{\mathrm{a}}$, Néstor Guerrero ${ }^{\mathrm{b}}$, \\ Maria Eugenia Marante ${ }^{\mathrm{b}}$, Julio Flórez-López ${ }^{\mathrm{c}}$ \\ ${ }^{a}$ Laboratoire de Mécanique et Technologie (LMT-Cachan) \\ Ecole Normale Supérieure de Cachan / CNRS-UMR 8535 / Université Paris 6 \\ 61 Avenue du Président Wilson, F-94235 Cachan Cedex, France \\ ${ }^{\mathrm{b}}$ Department of Structural Engineering, Lisandro Alvarado University \\ Barquisimeto, Venezuela \\ ${ }^{\mathrm{c}}$ Department of Structural Engineering \\ University of Los Andes Tulio Febres Codero, Mérida 5101, Venezuela
}

\begin{abstract}
It is proposed to identify the behavior of steel beams prior to and after the inception of local buckling by using Digital Image Correlation. Full field measurements are used to evaluate kinematic and static fields for determining constitutive laws. It enables for the detection of local buckling inception and the evaluation of the postbuckled behavior. Constitutive models are tuned by using measured Euler-Bernoulli kinematics.
\end{abstract}

Key words: Digital Image Correlation, Euler-Bernoulli kinematics, lumped damage mechanics, plastic hinge, steel beam 


\section{Introduction}

The description of the local behavior of steel beams in buildings, bridges, off-shore platforms or other civil engineering structures is desirable when designed against in-service or earthquake loadings. This task is performed by utilizing various numerical techniques. The finite element method [1] is one of the classical tools to analyze civil engineering structures. 2D $[2,3]$ or even 3D $[4,5]$ codes are run. Very detailed analyses are possible and require full 3D constitutive models. Simplified or semi-global analyses are another class of methods in which the kinematic fields are described by resorting to generalized degrees of freedom (e.g., displacements and section rotations in beams). The (non linear) constitutive laws are then integrated over the height of the element. Special, so-called multi-fiber, beam elements are then implemented [69]. Lumped damage mechanics (LDM) is another way to describe the local behavior of steel beams. It is based on an extension of the concept of plastic hinges in beam calculations. For instance, it is able to account for local buckling [10]. Up to now, the parameters of the constitutive law of hinges, and more generally of beams, relied upon global data such as displacements at few prescribed measurement points [11]. It is proposed to use full-field measurement techniques tailored for the description of these structures as a set of beams and joints to determine the constitutive behavior of steel beams.

Digital Image Correlation (DIC) is a photomechanical technique [12] allowing for the measurement of displacement fields based on a series of digital images of the surface of a specimen subjected to a specific loading history [13]. Recent advances have been achieved through a novel formulation that enables

\footnotetext{
* Corresponding author, Email: hild@lmt.ens-cachan.fr
} 
one to decompose the searched displacement field onto a suited library of such fields when dealing with beams (e.g., directly measuring the degrees of freedom associated with Euler-Bernoulli kinematics [14]). The interest of this experimental tool is that it provides full kinematic fields expressed in standard form for beam theories. Due to the fact that beam flexure is a statically determinate problem, static fields are also accessible when the behavior of the analyzed region is elastic. In particular, the exact loading conditions are obtained directly, and not inferred from the information given by the stroke and load transducers of the jack. The kinematics used herein is based upon an Euler-Bernoulli hypothesis applied to different discretizations of the region of interest. This hypothesis was also made in models, for instance developed by Sohal and Chen [15] to analyze local buckling of tubes by using inertia variations.

In the following, the procedure is illustrated by analyzing a flexural test on a steel beam. In Section 2, the general principle used in digital image correlation is briefly summarized and then particularized to an Euler-Bernoulli kinematics. A priori measurement uncertainties of the correlation algorithm are evaluated when applying a constant vertical displacement to the actual analyzed region. Kinematic and static fields are deduced by considering two different regions of interest in Section 3. Constitutive laws associated with local buckling are then determined by using two different descriptions (Section 4), namely, a global model in terms of generalized degrees of freedom and a non-local model accounting for inertia variations. 


\section{Kinematic measurements by DIC}

Figure 1a shows a reference picture of a cantilever beam loaded at its end section by an actuator. The beam has a length of $1.5 \mathrm{~m}$, a square cross-section of outer size $120 \mathrm{~mm}$ and inner size $112 \mathrm{~mm}$. It is made of a conventional construction steel (ASTM-A-500). The pictures were shot with a digital CMOS camera (resolution: $3888 \times 2592$ pixels, digitization: 8 bits). Figure 1 b corresponds to the last deformed shape of the considered beam. A random texture was applied onto the observed surface of the beam prior to the experiment to improve the measurement uncertainty by creating local gray level fluctuations.

\subsection{General principle}

DIC allows for the measurement of displacement fields from the registration (i.e., matching) of two pictures (Figure 1). The reference image, $f$ (gray level as a function of position $\boldsymbol{x}$ ), and that of the beam in a given deformed state $g$ are related by

$$
g(\boldsymbol{x}+\boldsymbol{u}(\boldsymbol{x}))=f(\boldsymbol{x})+b(\boldsymbol{x})
$$

where $b(\boldsymbol{x})$ is noise induced by image acquisition, and $\boldsymbol{u}(\boldsymbol{x})$ an in-plane displacement field. In the sequel, it is assumed that the noise level, $b$, is negligible either because of its low amplitude with respect to those of $f$ and $g$, or because of its scale separation with significant components of the displacement field. When $b()=$.0 , Equation (1) is the local form of the "brightness conservation." The problem to address is the identification of the displacement field $\boldsymbol{u}$ from the sole knowledge of pictures $f$ and $g$. As such, the problem is ill-posed, unless additional assumptions are made on the regularity of the displacement field so 
that the information is sufficient to determine $\boldsymbol{u}$ with a reasonable accuracy. Let us introduce the following objective functional $\Phi$ operating on a priori chosen displacement fields $v$

$$
\Phi^{2}(\boldsymbol{v})=\iint[g(\boldsymbol{x}+\boldsymbol{v}(\boldsymbol{x}))-f(\boldsymbol{x})]^{2} \mathrm{~d} \boldsymbol{x}
$$

In the present case, the trial displacement $\boldsymbol{v}$ will be chosen as that corresponding to an Euler-Bernoulli kinematics [14]. Functional $\Phi$ is non linear with respect to the unknown displacement field $\boldsymbol{u}$. Therefore, an iterative procedure is followed to evaluate $\boldsymbol{u}$. A Taylor expansion of $g$ up to the first order is introduced in Equation (2)

$$
\Phi_{\operatorname{lin}}^{2}(\boldsymbol{v})=\iint[g(\boldsymbol{x})-f(\boldsymbol{x})+\boldsymbol{v}(\boldsymbol{x}) \cdot \boldsymbol{\nabla} g(\boldsymbol{x})]^{2} \mathrm{~d} \boldsymbol{x}
$$

Equation (3) corresponds to the linearized correlation residuals associated with the brightness conservation. The displacement field is decomposed as a linear combination of basis functions $\boldsymbol{\eta}_{i}(\boldsymbol{x})$

$$
\boldsymbol{v}(\boldsymbol{x})=v_{i} \boldsymbol{\eta}_{i}(\boldsymbol{x})
$$

where Einstein's convention is used, so that $\Phi_{\text {lin }}^{2}$ is a quadratic form in the unknown amplitudes $v_{i}$. The extremality condition thus dictates, for all $j$,

$$
\left[\int\left(\boldsymbol{\nabla} g \cdot \boldsymbol{\eta}_{j}\right)(\boldsymbol{x})\left(\boldsymbol{\nabla} g \cdot \boldsymbol{\eta}_{k}\right)(\boldsymbol{x}) \mathrm{d} \boldsymbol{x}\right] v_{k}=\iint(f-g)(\boldsymbol{x})\left(\boldsymbol{\nabla} g \cdot \boldsymbol{\eta}_{j}\right)(\boldsymbol{x}) \mathrm{d} \boldsymbol{x}
$$

This system is written in a matrix form as

$$
[M]\{\check{v}\}=\{m\}
$$

where $\{\check{v}\}$ is a vector containing all the unknown components $v_{i},[M]$ and $\{m\}$ are known quantities dependent upon $f, g$, and $\boldsymbol{\eta}$. Successive iterations consist in evaluating the displacement field $\boldsymbol{v}$, correcting the deformed picture 
$g$ by using the current estimate $\boldsymbol{v}$ until no significant change of the degrees of freedom is observed. The evaluation of deformed images $g(\boldsymbol{x}+\boldsymbol{v}(\boldsymbol{x}))$ requires gray level interpolations, which in the present case are chosen to be bilinear.

\subsection{Euler-Bernoulli Kinematics: Beam-DIC}

In the following, the kinematics of a single beam is addressed. Let us consider a straight bar (i.e., a beam element) of uniform cross section and length $\ell$, which is loaded by axial forces and flexural moments about one axis of its cross section. Six degrees of freedom are considered for the bar, namely, two axial displacements $\left(v_{1}, v_{4}\right)$, two vertical displacements $\left(v_{2}, v_{5}\right)$ and two rotations $\left(v_{3}, v_{6}\right)$ about one axis of the cross section. The local coordinate system $(x, y, z)$ coincides with the principal axes of the cross section, with the $x$-axis representing the centroidal axis of the beam element, and the $z$-axis corresponding to the direction of the flexural moment.

The (chosen) displacement field in a beam element then reads

$$
\boldsymbol{v}(\boldsymbol{x})=\left[\boldsymbol{N}_{e}(\boldsymbol{x})\right]\{v\}
$$

where $N_{e}$ are the shape functions $\left(N_{1}, \ldots N_{6}\right)$ associated with the degrees of freedom $\{v\}^{t}=\left\{v_{1}, \ldots v_{6}\right\}$

$$
\begin{aligned}
& N_{1}(x)=1-\frac{x}{\ell} \quad, \quad N_{2}(x)=\left(2 x^{3}-3 \ell x^{2}+\ell^{3}\right) / \ell^{3} \\
& N_{3}(x)=\left(x^{3}-2 \ell x^{2}+\ell^{2} x\right) / \ell^{2} \quad, \quad N_{4}(x)=\frac{x}{\ell} \\
& N_{5}(x)=-\left(2 x^{3}-3 \ell x^{2}\right) / \ell^{3} \quad, \quad N_{6}(x)=\left(x^{3}-\ell x^{2}\right) / \ell^{2}
\end{aligned}
$$


The rotation field $\theta$ is only caused by flexure

$$
\theta(x)=\left[\frac{\mathrm{d} \hat{N}}{\mathrm{~d} x}(x)\right]\{\hat{v}\}
$$

where $\hat{v}^{t}=\left\{v_{2}, v_{3}, v_{5}, v_{6}\right\}$ and $[\hat{N}(x)]=\left[N_{2}(x), N_{3}(x), N_{5}(x), N_{6}(x)\right]$, and the corresponding curvature $\kappa$ reads

$$
\kappa(x)=\left[\frac{\mathrm{d}^{2} \hat{N}}{\mathrm{~d} x^{2}}(x)\right]\{\hat{v}\}
$$

Last, the longitudinal strain field is expressed as

$$
\varepsilon_{x x}(x, y)=\left[B_{e}(x, y)\right]\{v\}
$$

where $B_{e}=\left\{B_{1}, \ldots B_{6}\right\}$ reads

$$
\begin{aligned}
B_{1}(x, y)=-\frac{1}{\ell} & , & B_{2}(x, y)=-\frac{y}{\ell^{3}}(12 x-6 \ell) \\
B_{3}(x, y)=-\frac{y}{\ell^{2}}(6 x-4 \ell) & , & B_{4}(x, y)=\frac{1}{\ell} \\
B_{5}(x, y)=\frac{y}{\ell^{3}}(12 x-6 \ell) & , & B_{6}(x, y)=-\frac{y}{\ell^{2}}(6 x-2 \ell)
\end{aligned}
$$

Therefore, the generalized degrees of freedom $\{v\}$ give access to the displacement, rotation, curvature and strain fields at any point of the element without any additional post-processing step.

In practice, a region of interest (ROI) is chosen on the reference picture by the user. The ROI is then subdivided into smaller elements. The proposed approach is based upon first order Taylor expansions (3). When the (vertical) displacements are large, it will not be robust. An initial field is needed to start the calculation. A first evaluation of the vertical displacement field is obtained by performing a classical correlation analysis in which, for each correlation window, a uniform displacement is sought. An FFT algorithm is used and a sub-pixel value is determined by interpolating the correlation function in 
the vicinity of its maximum [16]. At least four windows are used so that the displacement field is interpolated by a cubic polynomial, or equivalently by using decomposition (7). Each pixel of the ROI is then moved by the estimated displacement. The subsequent displacement residuals are small so that the first order approximation used in Equation (3) is robust enough. The linear system (6) is implemented and solved. Convergence is reached when the correction of each measured degree of freedom $\{\tilde{v}\}^{t}=\left\{v_{1}, v_{2}, \ell v_{3}, v_{4}, v_{5}, \ell v_{6}\right\}$ is less than $10^{-3}$ pixel between two iterations.

\subsection{Performances of Beam-DIC}

One way of evaluating the performance of a correlation algorithm (i.e., displacement, rotation, curvature uncertainties) is to artificially move the ROI by translation increments of sub-pixel value, and to assess the standard uncertainty of measured quantities. By using the shift / modulation property of Fourier transforms [17], sub-pixel values are prescribed. In the present case, three quantities are evaluated, namely, the displacement, rotation and curvature fields. The same ROI (ROI 2 of Figure 1, size: $2048 \times 256$ pixels) is analyzed with different discretizations (i.e., the length of the element is varied, and its height is constant) and for a vertical displacement of 0.5 pixel. This last value generally leads to higher uncertainties when compared to other sub-pixel values [18].

Figure 2 shows the standard uncertainties for the three considered quantities. In the present case, the displacement uncertainty $\sigma_{u}$ is independent of the element length $\ell$ and reaches very low values (of the order of $10^{-3}$ pixel). This is due to the fact that many pixels are available to measure the displacement 
field described by very few degrees of freedom. The rotation uncertainty $\sigma_{\theta}$ decreases as the element size increases. Being the first derivative of the vertical displacement [see Equation (9)], this result is to be expected by dimensional analysis $\left(\sigma_{\theta} \propto \sigma_{u} / \ell\right)$. The linear fit shown in Figure 2 confirms this result. Similarly, the curvature uncertainty $\sigma_{\kappa}$ decays with the square of the element size. This is due to the fact that the curvature is the second derivative of the displacement field [see Equation (10)] and therefore $\sigma_{\kappa} \propto \sigma_{u} / \ell^{2}$ (this trend is depicted by the straight solid line in Figure 2). These results illustrate the fact that it is not possible to capture very fine kinematic details (i.e., displacement changes) with a high accuracy. There is always a trade-off between displacement uncertainty and spatial resolution (i.e., element size), provided the measured kinematics is well captured by the chosen kinematic basis [19].

\section{$3 \quad$ Kinematic and static fields}

The experiments are performed in such a way that the stroke of the jack is controlled. The applied force is also measured by a load cell close to the jack. Eleven pictures are shot, namely a reference picture and 10 load levels are recorded. Figure 3a shows the corresponding global data. Since the present situation is statically determinate, a first use of the correlation technique, ironically, will yield the change of the flexural moment along the longitudinal direction of the beam. This result is obtained by analyzing a ROI close to the load application point (ROI 1, see Figure 1a). In a second step, by analyzing a ROI containing the weld (ROI 2, see Figure 1a), the different fields introduced in Section 2.2 are evaluated prior to and after local buckling inception. 


\subsection{Elastic analysis: Load gauge}

The measured displacement field, and more importantly, the curvature field enable us to evaluate the flexural moment for any considered section of the beam (as for any classical load cell). This is possible provided the behavior of the region of interest remains elastic. In that sense, it is a "stress gauge" as already developed to analyze a Flamant problem [20]. Consequently, a first analysis consists in considering a ROI close to the zone where the load is applied (ROI 1, see Figure 1a). The chosen kinematics corresponds to the exact solution of a cantilever beam when the local behavior remains elastic. In the present case, a single element (height: 256 pixels, length: 2048 pixels) is considered. The advantage is that the measurement uncertainty is minimal (see Figure 2) and therefore the sought degrees of freedom are estimated more accurately. The curvature field then gives access to the flexural moment $M$ as a function of the local coordinate $x$

$$
\frac{M(x)}{E I_{0}}=\kappa(x)
$$

where $E$ is the Young's modulus of the material, and $I_{0}$ the inertia moment of the cross-sectional area. When the six degrees of freedom $\{v\}^{t}=\left\{v_{1}, \ldots v_{6}\right\}$ are measured, the curvature field is obtained directly by using Equation (10). Furthermore, the results are extrapolated outside the ROI by assuming that the beam behavior is elastic everywhere. This hypothesis is not necessarily true, and some deviations will be observed especially after local buckling inception.

Figure $3 \mathrm{~b}$ shows the change of the curvature field for the ten load levels. Two regimes are observed. For the first six levels, there is a gradual increase in the slope of the curvature. Thereafter, the latter levels off and decreases. This 
phenomenon is in direct relationship with the change of the applied load with the stroke (Figure 3a). The derivative of the curvature field is proportional to the applied load normal to the beam. In Figure 4a, the latter is compared with the force measured by the load cell. Even though there is a good correlation, a non-linear relationship exists between the two quantities. This first result shows that the closer the measurement region to the sample, the more relevant the information obtained by a transducer, be it a load cell or a "stress gauge" associated with a Beam-DIC procedure.

Two other outputs of this first analysis are given. First, the location of the point for which the flexural moment vanishes is sought. Figure $4 \mathrm{~b}$ shows the change of the axial location of that point with the applied load. There is a general correlation between the two data. However, the location clearly moves and neglecting this effect would over-estimate the value of the flexural moment by a factor up to about $10 \%$. The average correlation residual is shown in Figure 5 for the ten analyzed pictures. Even though there is a gradual degradation of its level, the order of magnitude remains the same. Therefore, the previous measurements are deemed trustworthy.

\subsection{Hinge detection}

The rotation and deflection fields are analyzed in the sequel. One of the features that will be analyzed concerns the results at the junction of the beam with its base. This point corresponds to the location of the weld. Figure 6a shows the change of the rotation field for the ten analyzed pictures. Two regimes are observed. For the first five pictures, the rotation level close to the weld is vanishingly small. Conversely, for the five last levels, there is a 
discontinuity of the extrapolated rotation at the junction. This phenomenon corresponds to the effect of local buckling that is not fully captured by the chosen kinematics. The displacement field is shown in Figure 6b. Again two regimes are observed. The extrapolated displacement close to the junction is first very small, i.e., continuous with respect to the kinematics of the base. Later on, a deviation is observed and the point for which the extrapolated displacement vanishes is shifted about 200 pixels away from the junction.

\subsection{Kinematic field: Analysis of local buckling}

The considered ROI is now moved close to the junction (ROI 2, see Figure 1a) to analyze local buckling. The left edge of the ROI now coincides with the estimated location of the junction (i.e., weld) between the beam and the base. To discuss the choice of the discretization used hereafter, the last loading case is first analyzed; local buckling has already occurred. First, only one element is chosen and the kinematic field is sought. By analyzing the correlation residual field (Figure 7), there is a clear deviation from the average level for the first 250 pixels. This is an indication that the measurement results in that zone are not reliable. The main reason is that the kinematic hypothesis is not consistent with the experimental phenomenon (i.e., local buckling) in this localized region. When studying the rotation field (Figure 8a), a discontinuity is again observed as in the case of extrapolated quantities of Section 3.1.

The reason for the observed discrepancy between measured and actual displacements is mainly due to the fact that the chosen (Euler-Bernoulli) kinematics (with uniform elastic and geometric properties) is not able to properly describe the phenomena occurring close to the junction. One alternative is 
then to only use measurements outside of this zone, as shown in Section 3.1, and extrapolate the results up to the junction. Another choice is to resort to more detailed analyses of the local field by using stereovision $[21,22]$ since out of plane motions may cause in-plane distorsions. It was followed to analyze buckling in composite materials [23]. A last choice is to still use Euler-Bernoulli kinematics, but not with the usual constraints related to the assumption of elastic behavior. The last route is followed herein. It consists in discretizing the ROI by using more than one element. Consequently, more degrees of freedom are measured, i.e., the displacement field is richer. However, the measured displacement field does not necessarily satisfy static admissibility. The latter is recovered when the curvature and its derivative are continuous from one element to the next one. Let us emphasize that the correlation algorithm only enforces continuities of the displacements and rotations thanks to the chosen degrees of freedom. The curvature, derivative of the curvature and normal strains are not necessarily continuous functions when more than one beam element is considered in the ROI.

In the following, the measured displacement field will be regularized by enforcing the continuity of the curvature field over the whole ROI. Moreover, a continuity of the rotation and displacement at the weld is prescribed for a discretization with more than two elements. This is possible by measuring the rigid body motion of the base with the same type of correlation scheme as that discussed in Section 2.1. Last, over one part of the ROI where elasticity is likely to occur, additional constraints are prescribed to describe the continuity of the curvature derivative and normal strains. The regularization of any quantity is performed by using Lagrange multipliers. All the choices discussed above have to be validated. 
The performance of the correlation algorithm is evaluated by following the same procedure as in Section 2.3, namely, for a constant vertical displacement of 0.5 pixel. In Figure 2, the results show that the same level of uncertainty is achieved for displacements, rotations and curvature, irrespective of the element size $\ell$. This is to be expected since the additional constraints lead to an equivalence of any discretization when the continuity of the curvature, its first derivative and the normal strains are continuous over the whole length of the ROI. The fact that this property is recovered validates the implemented regularization. Last, when only the curvature is constant over the whole ROI, the displacement uncertainty level is virtually constant. The rotation uncertainty decays approximately with the square root of the element size $\left(\sigma_{\theta} \propto 1 / \sqrt{\ell}\right)$, and the curvature with the element size raised to the power $-1.2\left(\sigma_{\kappa} \propto 1 / \ell^{1.2}\right)$. Both uncertainties reach the same level as in the two other analyzed cases when the element length is equal to 2048 pixels. Therefore, an intermediate response is observed between the two limit cases analyzed previously. These last results are not shown in Figure 2 for the sake of clarity. It is concluded that the higher the number of implemented continuity conditions, the less uncertain the measurements, provided the kinematic basis contain the field to be evaluated.

The first analysis concerns the choice of the discretization when the displacement field is not "regularized" over the whole length of the ROI. This is a crucial step since the smaller the element, the better the description of a complex displacement field, yet the higher the uncertainty level (Figure 2). Figure 8b shows the rotation field when the ROI is discretized with 1 to 16 elements. When less than two elements are used, no regularization is implemented. The consequence is that the rotation at the left end of the ROI is not equal to the 
value corresponding to the rotation of the base. Moreover, the derivative of the rotation (i.e., the curvature) is not continuous at the middle of the ROI when two elements are used. When 4 or 8 elements are used, virtually the same rotation field is obtained. Last, when 16 elements are used, fluctuations occur, thereby indicating that the evaluation is no longer trustworthy. When the correlation residuals are analyzed (Figure 9), there is a continuous decrease of the average residual over the whole ROI. Conversely, there is a slight increase when the average is performed over the first 256 pixels for 4 elements. This effect is due to the regularization that is performed starting with four elements. The average residual then decreases again. Both averages level off for discretizations with more than 8 elements when the curves showing the trends are studied. Consequently, the number of elements is chosen equal to 8 elements in all subsequent analyses to limit spurious fluctuations.

Another question to address concerns the zone where the regularization is applied. On the one hand, if the zone is too large, a deviation is to be expected. On the other hand, if the zone is too small, the measurements might be noisier. In the present case, 8 elements are considered and different lengths of the right part of the ROI are regularized, namely, the curvature, its derivative and the normal strains are continuous. In that part, it is assumed that the beam behavior is elastic. Over this zone, it would be equivalent of using a single Euler-Bernoulli beam element. Figure 10a shows the measured rotation field as a function of the fraction of regularized ROI. The results are very close when up to $75 \%$ of the ROI is regularized. Beyond this value, there is a clear change of the measured rotation field. The corresponding correlation residuals also start to increase, especially in the left part of the beam. In the following, a regularization over $75 \%$ of the $\mathrm{ROI}$ is chosen. 
The rotation field is now studied for the ten analyzed load levels (Figure 10b). As the stroke increases, a gradual deviation from an elastic response is observed. For the last four levels, there is a rotation localization induced by local buckling. This result illustrates the fact that the rotation capacity of steel beams is undermined by the inception and development of local buckling. The measured rotation fields are now used to identify different constitutive laws. To perform the analysis, two inputs are needed, namely, the rotation field and extrapolated curvature field in an elastic region, which is proportional to the flexural moment.

\section{Constitutive law of a beam subject to local buckling}

The previous measurements are now used to identify two different constitutive equations depending on the type of hypothesis made to describe local buckling.

\subsection{First model: Lumped constitutive law}

If the experiments are performed in order to analyze the beam behavior when used in frames, a simple yet powerful model consists in "lumping" all the non-linear processes induced by plasticity and local buckling onto the weld section. This type of assumption is made in the framework of Lumped Damage Mechanics [24-26]. It corresponds to an extension of the concept of plastic hinges for which constitutive laws describe the flexural moment / section rotation relationship. This analysis is possible in the present case by using the extrapolated rotation (Figure 6a) and the corresponding curvature fields (Figure $3 \mathrm{~b}$ ). For the section rotation at the weld, the value of the base rotation is 
subtracted from its extrapolated value. The corresponding flexural moment is proportional to the value of the extrapolated curvature field at the location of the weld.

Figure 11 shows the results obtained with the present procedure. Two regimes are observed. For the first five load steps, the rotation level is very small and believed to be in the uncertainty range associated with the extrapolation procedure. Conversely, for the last five load levels, there is a clear change of the rotation level induced by local buckling. With this type of information, there is no clear indication of a plastic regime prior to local buckling. However, it clearly shows the degradation of the rotation capacity of steel beams induced by local buckling. When compared with Figure 3a, there is a significant difference in terms of behavior, in particular for the load levels where local buckling occurs.

In more quantitative terms, the following model can be used to describe the loading part of the experiment. The elastic part is such that

$$
M=E I_{0} \kappa \text { and } \Theta=0
$$

where $\Theta$ denotes the section rotation at the clamp. These relationships are true as long as $M<M_{u}, M_{u}$ being the ultimate flexural moment. When the flexural moment becomes equal to $M_{u}$, a plastic hinge develops due to local buckling. Beyond a critical angle $\Theta_{0}$, softening occurs. This last part of the behavior is described by a damage variable $D$ lumped at the clamp

$$
M=M_{u}(1-D) \text { when } \Theta>0
$$

with

$$
D=\Delta\left\langle\Theta-\Theta_{0}\right\rangle
$$


where $\langle$.$\rangle are Macauley's brackets. By using a least squares fit, the following$ values are determined: $M_{u} / E I_{0}=26.2 \times 10^{-6} \pm 0.4 \times 10^{-6}$ pixel $^{-1}, \Theta_{0}=$ $0.02 \pm 0.004$, and $\Delta=9 \pm 4$. The identification result is shown in Figure 11. The lumped model is able to capture the main features observed experimentally.

\subsection{Second model: Piece-wise constant inertia}

In the present approach, it is assumed that local buckling is described by a change of the beam inertia, to be determined, over an unknown length $\ell_{l b}$ close to the weld (Figure 12). An Euler-Bernoulli hypothesis is made to analyze the experiments. It is assumed that the rotation and curvature fields are both continuous, in a similar way as during the measurement stage. Some of these hypotheses can also be found in Refs. [15,27]. In the first part of the beam

where the inertia is denoted by $\tilde{I} \leq I_{0}$ (i.e., $0 \leq x \leq \ell_{l b}$ ), the rotation field reads

$$
\theta(x)=\frac{P}{E \tilde{I}}\left(L_{p} x-\frac{x^{2}}{2}\right)+\theta_{0}
$$

where $\theta_{0}$ denotes the rotation of the base, and $L_{p}$ the length leading to a continuous curvature field. In the second part $\left(x>\ell_{l b}\right)$, the rotation field becomes

$$
\theta(x)=\frac{P}{E I_{0}}\left[L\left(x-\ell_{l b}\right)-\frac{x^{2}-\ell_{l b}^{2}}{2}\right]+\frac{P}{E \tilde{I}}\left(L_{p} \ell_{l b}-\frac{\ell_{l b}^{2}}{2}\right)+\theta_{0}
$$

where the continuity of the curvature when $x=\ell_{l b}$ yields the additional constraint

$$
\frac{L-\ell_{l b}}{I_{0}}=\frac{L_{p}-\ell_{l b}}{\tilde{I}}
$$

For each load level, two unknowns $\left(\tilde{I}\right.$ and $\left.\ell_{l b}\right)$ are tuned by least squares minimization between the measured rotation field (Figure 10b) and that described 
by Equation (18). The length $L$ is deduced from the static analysis of Section 3.1, see Figure $4 \mathrm{~b}$, and the value $P / E I_{0}$ from the analysis of the rotation in the zone where it is assumed to be elastic. In the present case, the value for $\ell_{l b}$ varies from $0.26 L_{R O I}$ for the fifth load level to $0.24 L_{R O I}$ for the last one, where $L_{R O I}$ denotes the length of the region of interest (here 2048 pixels). This last value is assumed to be valid for the whole analysis, and is about twice the distance of the skeletal point shown in Figure 6b.

Figure 13a shows the results obtained with the present procedure. There is a very good agreement between the measured and identified rotation fields. The results obtained so far are recast as a non-local damage model for which the inertia degradation is directly described by a damage parameter $\tilde{D}$

$$
\tilde{I}=I_{0}(1-\tilde{D})
$$

so that when $\tilde{D}=0$, there is no buckling, and when $\tilde{D}=1$, the rotation capacity of the beam is completely degraded. The quantity $\ell_{l b}$ is then the "internal length" of the non-local model, here assumed to be constant. Within this new framework, it is also possible to identify the change of the damage parameter $D$ with the average curvature $\tilde{\kappa}$ over the length $\ell_{l b}$ (Figure $13 \mathrm{~b}$ ). Moreover, the following law is used to fit the data

$$
\tilde{D}=1-\exp \left[-\left(\frac{\left\langle\tilde{\kappa}-\kappa_{t h}\right\rangle}{\kappa_{0}}\right)^{m}\right]
$$

where $\kappa_{0}$ is a scale parameter, and $\kappa_{t h}$ a threshold value below which no degradation (i.e., local buckling) occurs. A very good description of the experimental data is obtained by using this expression when $\kappa_{0}=2.2 \times 10^{-6} \pm$ $10^{-6} 1 /$ pixel, $\kappa_{t h}=1.9 \times 10^{-5} \pm 2 \times 10^{-6} 1 /$ pixel, and $m=0.34 \pm 0.05$. The latter ones were determined by resorting to a least squares fit. 


\section{Summary}

The analysis of Euler-Bernoulli displacement fields based on digital image correlation is used to identify constitutive models of steel beams prior and after the inception of local buckling. When the local behavior is elastic, it allows one to measure the flexural moment. Otherwise, this kinematics is able to describe local buckling, in particular in terms of rotation field. With this information at hand, two models are identified. The first one corresponds to a "lumped" point of view that may be used to model frames within the framework of lumped damage mechanics. The second model is non-local for which the growth law of the damage variable is determined, as well as the internal length, which is virtually constant during the whole development of local buckling.

The definition of a suited library (i.e., with mechanical content as close as possible to the real experiment) of displacement field reveals to be a key ingredient for an accurate and reliable evaluation of the beam kinematics in terms of generalized degrees of freedom. When applied to a real experiment, the feasibility is demonstrated by using pictures obtained with a semi-professional camera. It is also shown that when one departs from the kinematic hypotheses made herein, the correlation residuals are a good error indicator. In present case, it allows for the detection of local buckling and its effect on the rotation capacity of steel beams. By moving artificially pictures, a displacement uncertainty of the order of one $\mu \mathrm{m}$ is achieved with the chosen parameters.

The same approach can be applied to other types of structures such as frames, or other types of materials such as reinforced concrete or wood. When de- 
parting from beam kinematics, the approach developed herein is generic and is applicable to cases where closed-form solutions exist $[28,29]$, or numerical solutions [30] are computed. The key ingredient is to taylor the measured kinematics as closely as possible to the experiment, and to the (non linear) constitutive law.

\section{Acknowledgements}

This work is part of an ECOS-Nord / CNRS project entitled "Detection and prediction of the damage state in frames subjected to earthquakes." 


\section{References}

[1] O. C. Zienkievicz and R. L. Taylor, The Finite Element Method, (McGraw-Hill, London (UK), 4th edition, 1989).

[2] H. G. Kwak and F. C. Filippou, Nonlinear FE analysis of R/C structures under monotonic loads, Comput. Struct. 65 [1] (1997) 1-16.

[3] N. Ile, J. M. Reynouard and J. F. Georgin, Non-linear response and modelling of RC walls subjected to seismic loading, ISET J. Earthqu. Technol. 39 [1-2] (2002) 2002.

[4] J. Y. R. Rashid, R. A. Dameron and R. S. Dunham, Finite element analysis of reinforced concrete in bridge seismic design practice, in: Modeling of inelastic behavior of RC structures under seismic loads, P. B. Shing and T. Tanabé, eds., (ASCE, 2001), 217-233.

[5] M. Kwon and E. Spacone, Three-dimensional finite element analyses of reinforced concrete columns, Comput. Struct. 80 [2] (2002) 199-212.

[6] D. R. J. Owen and E. Hinton, Finite elements in plasticity: Theory and practice, (Pineridge Press Ltd, Swansea, UK, 1980).

[7] J. Guedes, P. Pégon and A. Pinto, A fiber Timoshenko beal element in CASTEM 2000, (JRC, I-21020, Ispra, Italy, Special publication No. I.94.31, 1994).

[8] L. Davenne, F. Ragueneau, J. Mazars and A. Ibrahimbegovic, Efficient approaches to finite element analysis in earthquake engineering, Comput. Struct. 81 [12] (2003) 1223-1239.

[9] P. Kotronis and J. Mazars, Simplified Modelling Strategies to Simulate the Dynamic Behaviour of R/C Walls, J. Earthquake Eng. 9 [2] (2005) 285-306. 
[10] N. Guerrero, M. E. Marante, R. Picón and J. Flórez-López, Model of local buckling in steel hollow structural elements subjected to biaxial bending, $J$. Construct. Steel Res. 63 [6] (2007) 779-790.

[11] M. E. Marante and J. Flórez-López, Three-dimensional analysis of reinforced concrete frames based on lumped damage mechanics, Int. J. Solids Struct. 40 [19] (2003) 5109-5023.

[12] P. K. Rastogi, edts., Photomechanics, (Springer, Berlin (Germany), 2000), 77.

[13] T. C. Chu, W. F. Ranson, M. A. Sutton and W. H. Petters, Applications of Digital-Image-Correlation Techniques to Experimental Mechanics, Exp. Mech. 3 [25] (1985) 232-244.

[14] F. Hild, S. Roux, R. Gras, N. Guerrero, M. E. Marante and J. Flórez-López, Displacement Measurement Technique for Euler-Bernoulli Kinematics, Opt. Lasers Eng. 47 (2009) 495-503.

[15] I. S. Sohal and W.-F. Chen, Local buckling and sectional behavior of fabricated tubes, ASCE J. Struct. Eng. 113 [3] (1987) 519-533.

[16] F. Hild, B. Raka, M. Baudequin, S. Roux and F. Cantelaube, Multi-Scale Displacement Field Measurements of Compressed Mineral Wool Samples by Digital Image Correlation, Appl. Optics IP 41 [32] (2002) 6815-6828.

[17] J. N. Périé, S. Calloch, C. Cluzel and F. Hild, Analysis of a Multiaxial Test on a C/C Composite by Using Digital Image Correlation and a Damage Model, Exp. Mech. 42 [3] (2002) 318-328.

[18] G. Besnard, F. Hild and S. Roux, "Finite-element" displacement fields analysis from digital images: Application to Portevin-Le Châtelier bands, Exp. Mech. 46 (2006) 789-803.

[19] J. Réthoré, F. Hild and S. Roux, Extended digital image correlation with crack shape optimization, Int. J. Num. Meth. Eng. 73 [2] (2008) 248-272. 
[20] S. Roux, F. Hild and S. Pagano, A stress scale in full-field identification procedures: A diffuse stress gauge, Eur. J. Mech. A/Solids 24 (2005) 442-451.

[21] M. A. Sutton, S. R. McNeill, J. D. Helm and Y. J. Chao, Advances in TwoDimensional and Three-Dimensional Computer Vision, in: Photomechanics, P. K. Rastogi, eds., (Springer, Berlin (Germany), 2000), 323-372.

[22] D. Garcia, J.-J. Orteu and L. Penazzi, A combined temporal tracking and stereo-correlation technique for accurate measurement of 3D displacements: Application to sheet metal forming, J. Mat. Proc. Tech. 125-126 (2002) 736742.

[23] L. G. Melin and J. Schön, Buckling behaviour and delamination growth in impacted composite specimens under fatigue load: An experimental study, Comp. Sci. Tech. 61 (2001) 1841-1852.

[24] A. Cipollina, A. López-Inojosa and J. Flórez-López, A simplified damage mechanics approach to nonlinear analysis of frames, Comput. Struct. 54 [6] (1995) 1113-1126.

[25] M. E. Marante and J. Flórez-López, model of damage for RC elements subjected to biaxial bending, Eng. Struct. 24 [9] (2002) 1141-1152.

[26] F. Armero and D. Ehrlich, Numerical modeling of softening hinges in thin Euler-Bernoulli beams, Comput. Struct. 84 [10-11] (2006) 641-656.

[27] N. Challamel and M. Hjiaj, Non-local behavior of plastic softening beams, Acta Mech. 178 (2005) 125-146.

[28] F. Hild and S. Roux, Digital image correlation: From measurement to identification of elastic properties - A review, Strain 42 (2006) 69-80.

[29] S. Roux and F. Hild, Stress intensity factor measurements from digital image correlation: Post-processing and integrated approaches, Int. J. Fract. 140 [1-4] (2006) 141-157. 
[30] H. Leclerc, J.-N. Périé, S. Roux and F. Hild, Integrated Digital Image Correlation for the Identification of Mechanical Properties, in: MIRAGE 2009, A. Gagalowicz and W. Philips, eds., (Springer-Verlag, Berlin (Germany), 2009), LNCS 5496 161-171. 


\section{List of Figures}

1 Cantilever beam in its reference (a) and deformed (b) state for the last load level. The two regions of interest (ROIs) analyzed herein are shown on the reference picture. The picture resolution is $3888 \times 2592$ pixels with an 8 -bit digitization. The physical size of 1 pixel is $0.39 \mathrm{~mm}$.

2 Displacement, rotation and curvature standard uncertainties as functions of the element length $\ell$ for a vertical displacement of 0.5 pixel of ROI 2 of Figure 1. Two correlation algorithms are compared. The first one (solid line and solid symbols) is a direct analysis with Euler-Bernoulli kinematics. The second one (dashed lines and open symbols) is a regularized algorithm for which a continuity of the curvature, its derivative and the normal strain is enforced.

3 Applied load versus stroke for the analyzed experiment (a). Each measurement point is labeled by a letter. Curvature fields for the ten applied load levels analyzed herein (b). The location of ROI 1 is shown and the fields are extrapolated outside the ROI by assuming an elastic behavior.

4 Derivative of the curvature vs. measured load (a). Location of the point where the flexural moment vanishes vs. measured $\operatorname{load}(\mathrm{b})$. 
5 Average correlation residuals $\Phi$ as a function of the applied load.

6 Rotation fields for the ten load levels (a). The location of ROI 1 is shown and the fields are extrapolated outside the ROI by assuming an elastic behavior. The location of the weld is also depicted by the dashed line on the left. Deflection fields for the ten load levels (b). The location of ROI 1 is shown and the fields are extrapolated outside the ROI by assuming an elastic behavior.

7 Residual field for the last load level for ROI 2 containing the zone where local buckling occurs.

8 Rotation field for the last load level (a). A rotation discontinuity is observed on the left end of the ROI. The location of the weld is depicted by the dashed line. Rotation field for the last load level when different numbers of elements are used (b). When at least four elements are used, the measurement is regularized on $75 \%$ of the beam length and the base rotation is prescribed on the left part of the ROI.

9 Average residuals (in gray levels) as a function of number of beam elements. Two zones are considered, the first 256 pixels and the whole 2048-pixel ROI to evaluate the average value. The curves depict trends in terms of change of the correlation residual with the chosen discretization. 
10 Rotation field for the last load level when different percentages of the right part of the ROI length are regularized (a). Rotation fields for the ten analyzed load levels (b). The dashed line depicts the location where the regularization starts.

11 Normalized flexural moment $M / E I_{0}$ vs. corrected section rotation $\Theta$ for the analyzed experiment. The dashed line shows the identification result with the lumped model [Equations (14-16)].

12 Beam with a degraded inertia $\tilde{I}$ over a length $\ell_{l b}$ with respect to a reference inertia $I_{0}$.

13 Rotation fields for the ten analyzed load levels (b). Comparison between experimental and predicted rotations when a constant inertia degradation is assumed in the left part of the beam (b). Damage parameter $\tilde{D}$ vs. average curvature $\tilde{\kappa}$. The dashed line corresponds to the best fit with the damage law (21). 


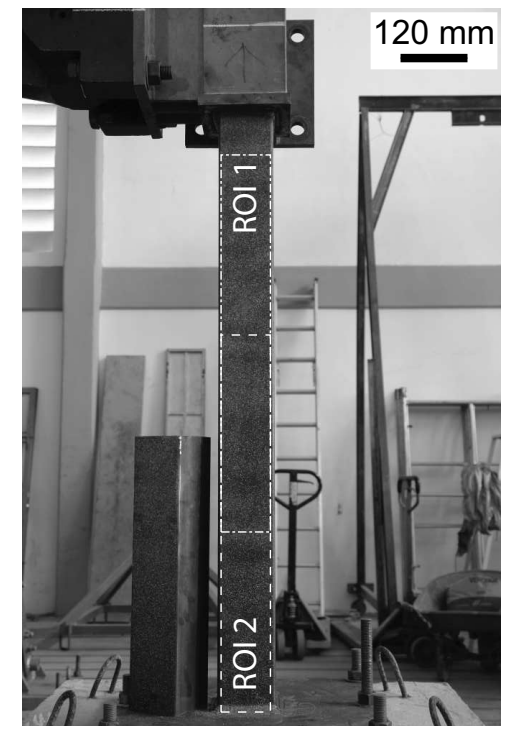

(a)

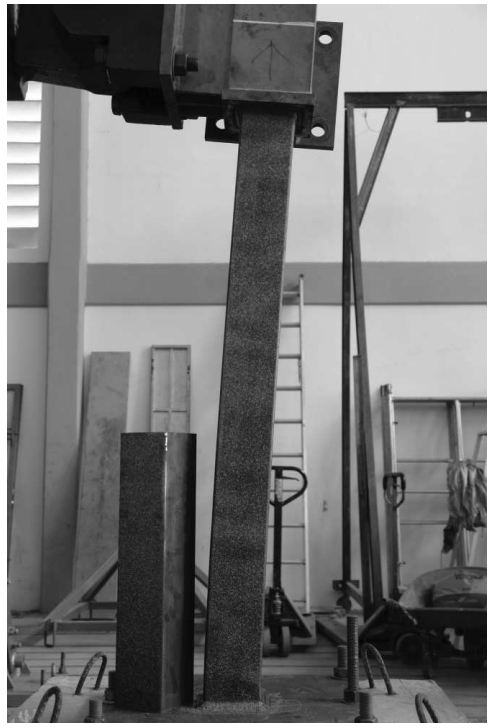

(b)

Fig. 1. Cantilever beam in its reference (a) and deformed (b) state for the last load level. The two regions of interest (ROIs) analyzed herein are shown on the reference picture. The picture resolution is $3888 \times 2592$ pixels with an 8 -bit digitization. The physical size of 1 pixel is $0.39 \mathrm{~mm}$. 


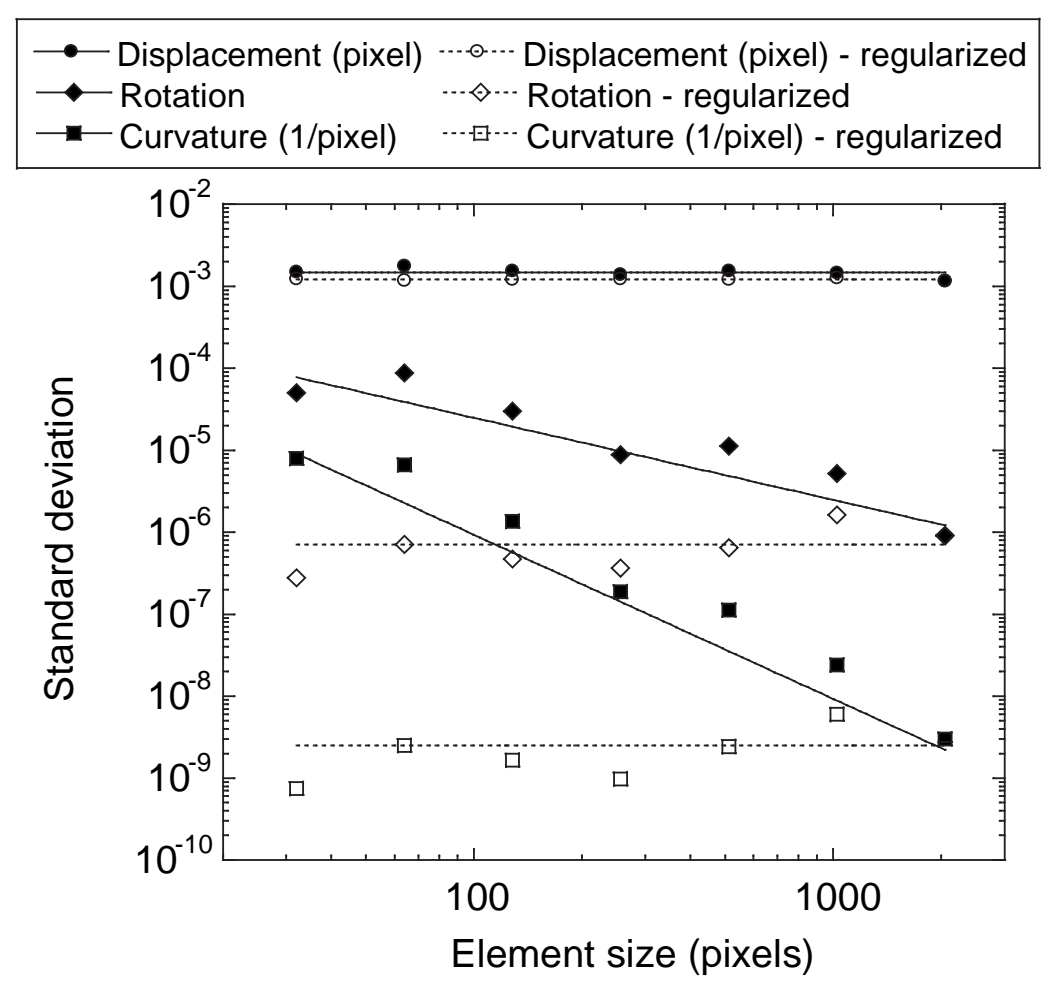

Fig. 2. Displacement, rotation and curvature standard uncertainties as functions of the element length $\ell$ for a vertical displacement of 0.5 pixel of ROI 2 of Figure 1 . Two correlation algorithms are compared. The first one (solid line and solid symbols) is a direct analysis with Euler-Bernoulli kinematics. The second one (dashed lines and open symbols) is a regularized algorithm for which a continuity of the curvature, its derivative and the normal strain is enforced. 


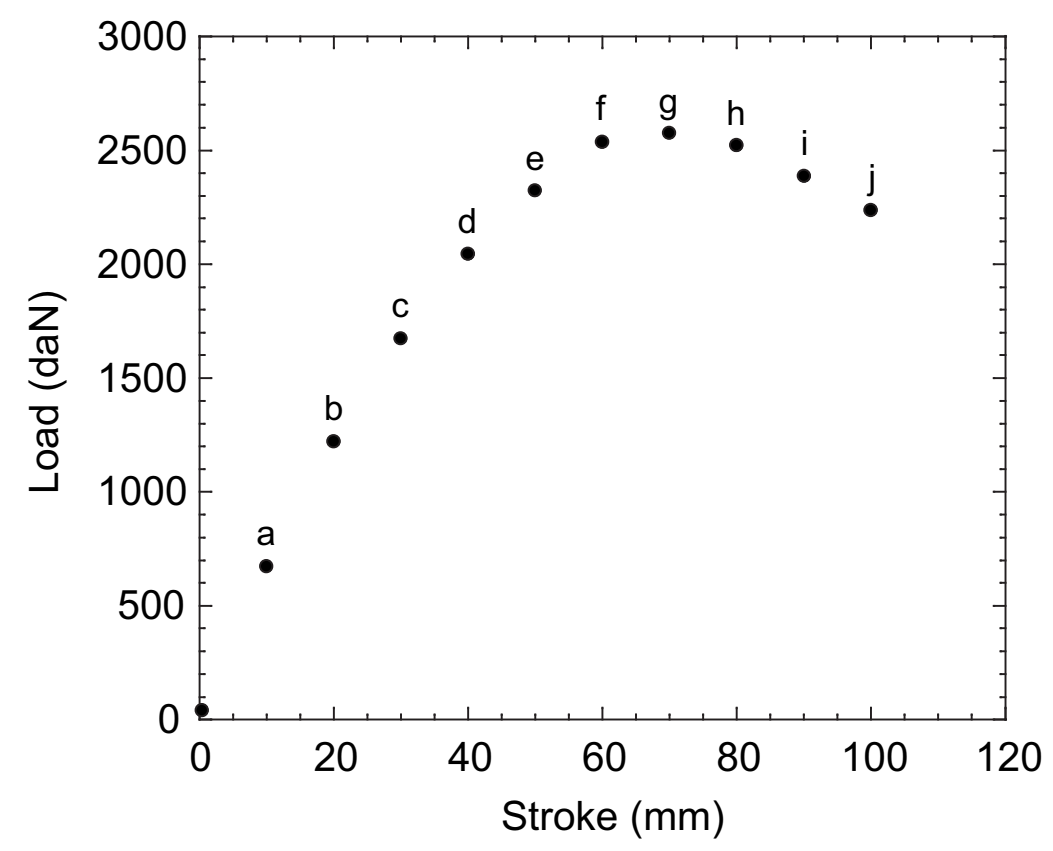

(a)

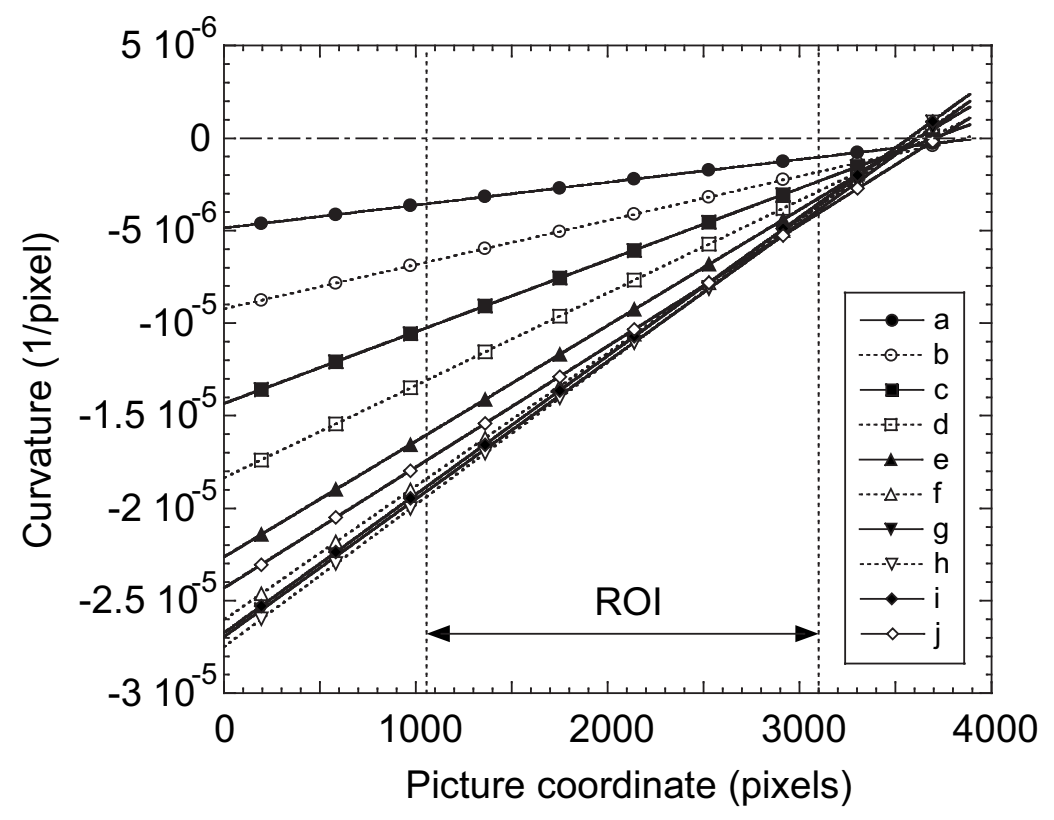

(b)

Fig. 3. Applied load versus stroke for the analyzed experiment (a). Each measurement point is labeled by a letter. Curvature fields for the ten applied load levels analyzed herein (b). The location of ROI 1 is shown and the fields are extrapolated outside the ROI by assuming an elastic behavior. 




(a)

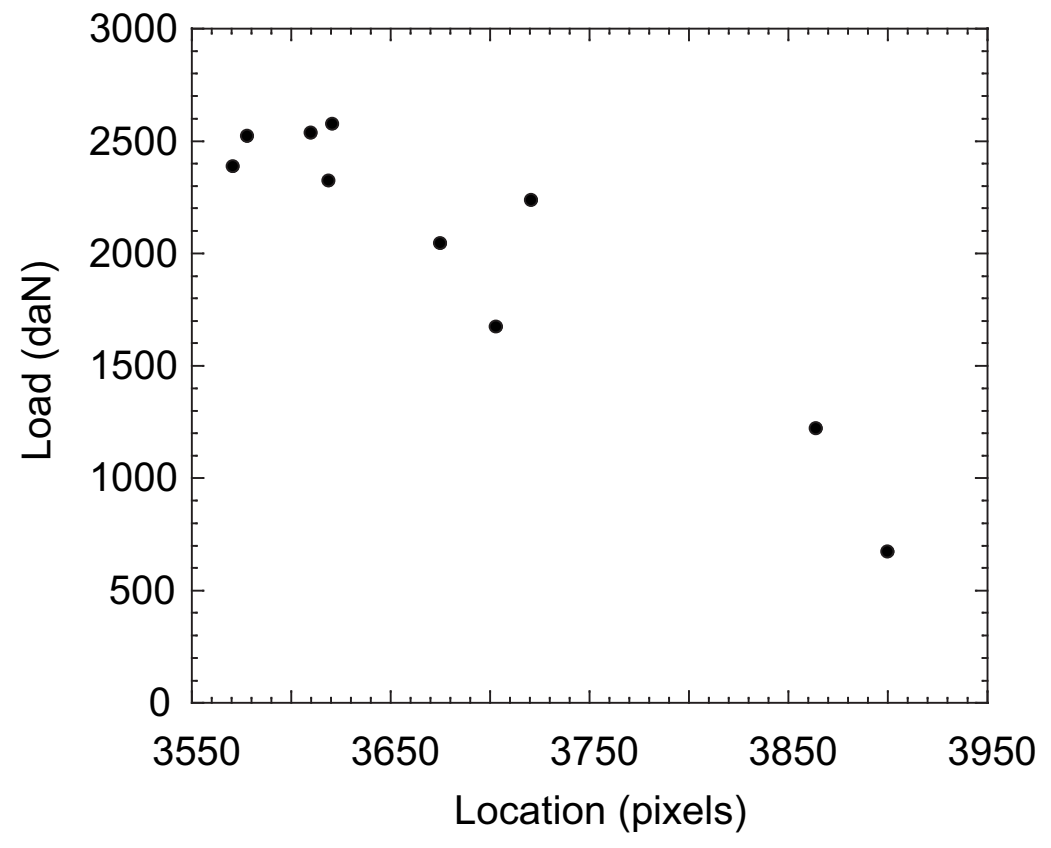

(b)

Fig. 4. Derivative of the curvature vs. measured load (a). Location of the point where the flexural moment vanishes vs. measured load (b). 




Fig. 5. Average correlation residuals $\Phi$ as a function of the applied load. 


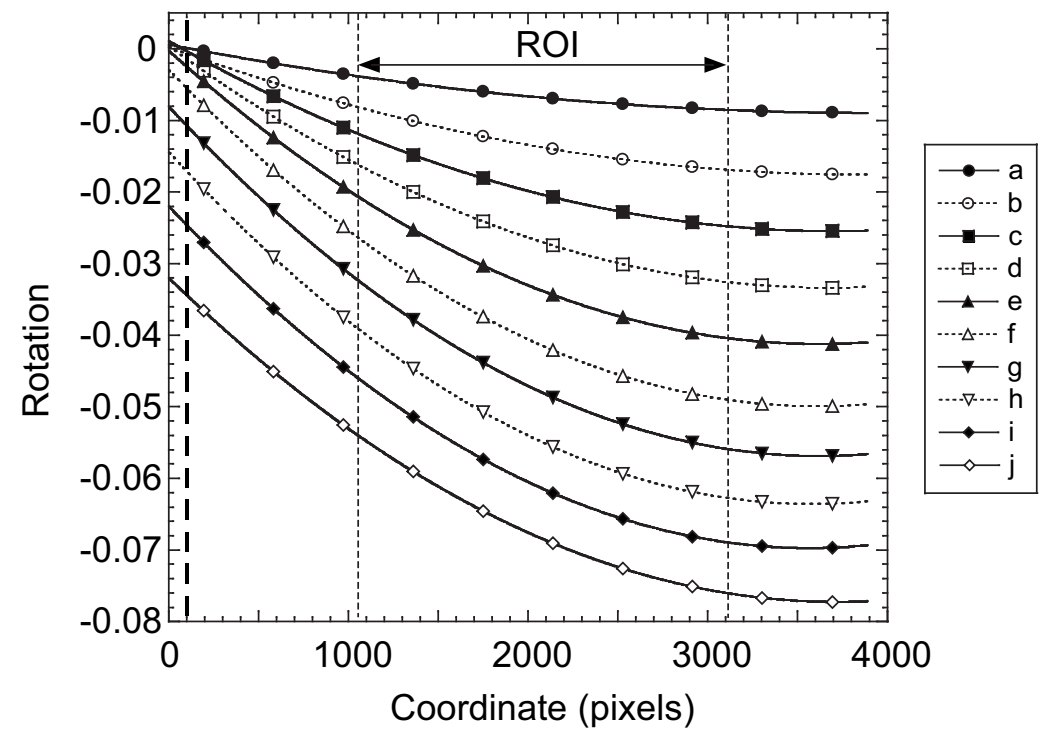

(a)

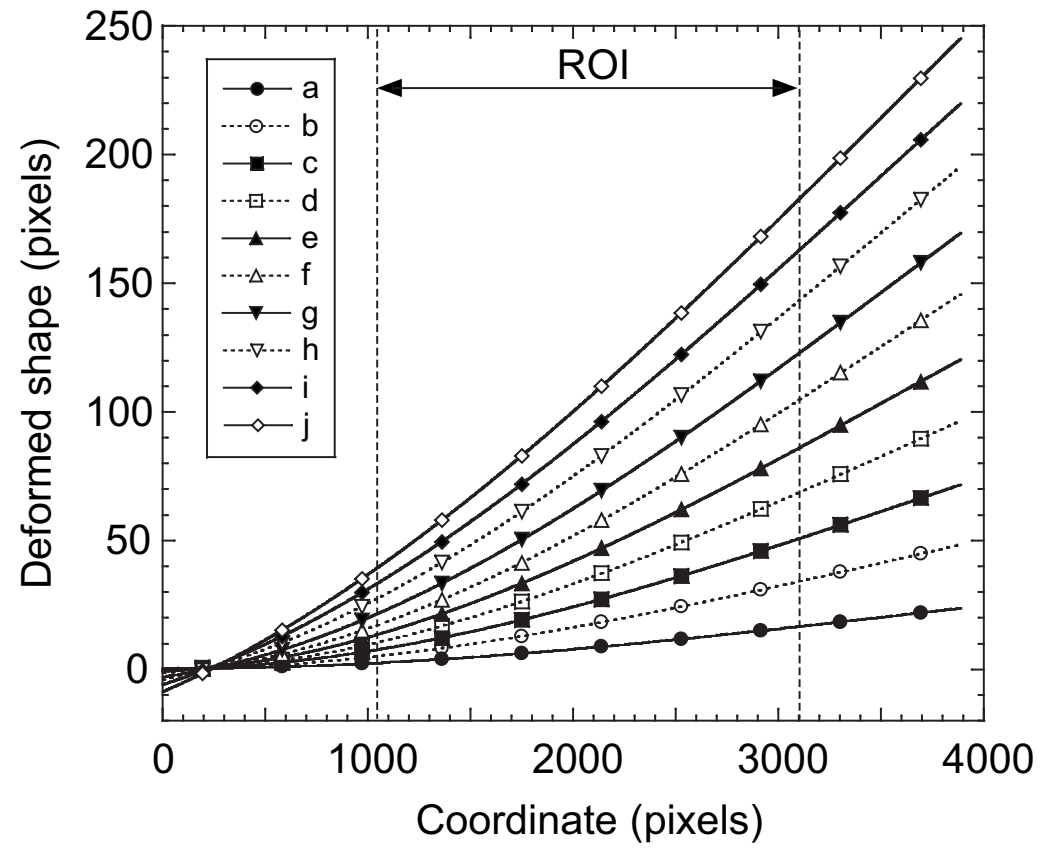

(b)

Fig. 6. Rotation fields for the ten load levels (a). The location of ROI 1 is shown and the fields are extrapolated outside the ROI by assuming an elastic behavior. The location of the weld is also depicted by the dashed line on the left. Deflection fields for the ten load levels (b). The location of ROI 1 is shown and the fields are extrapolated outside the ROI by assuming an elastic behavior. 


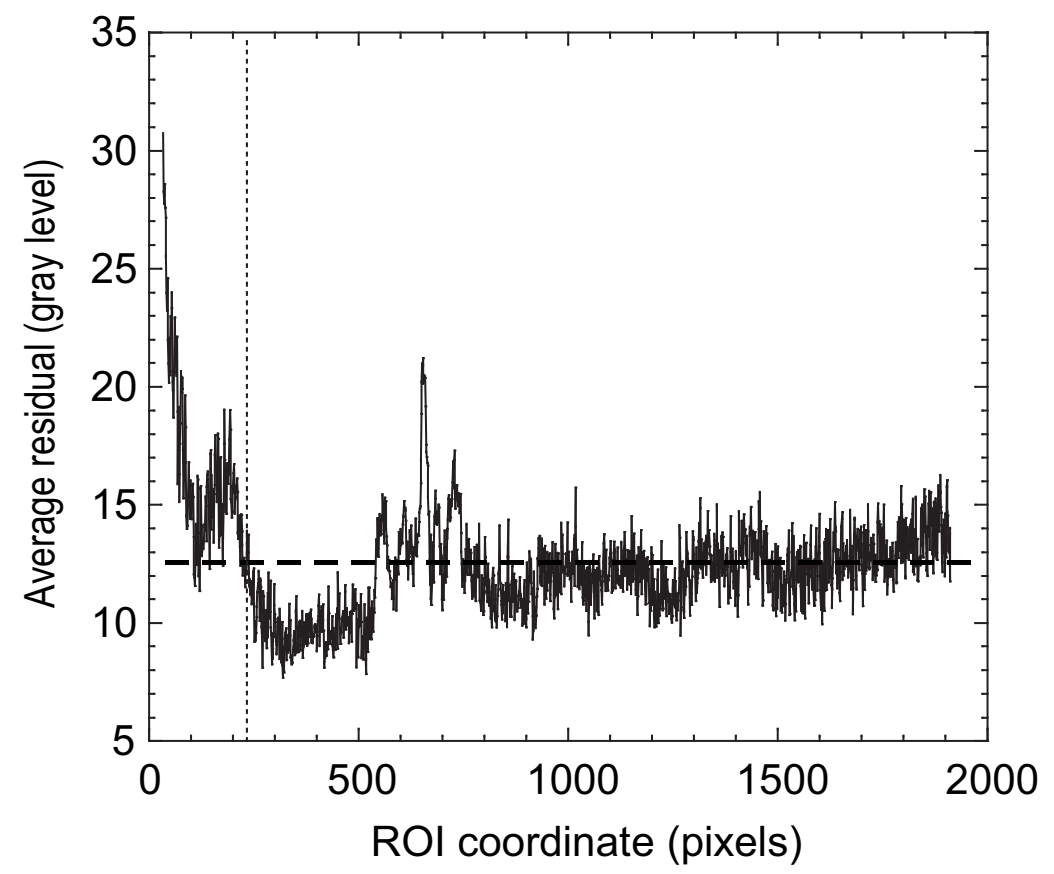

Fig. 7. Residual field for the last load level for ROI 2 containing the zone where local buckling occurs. 


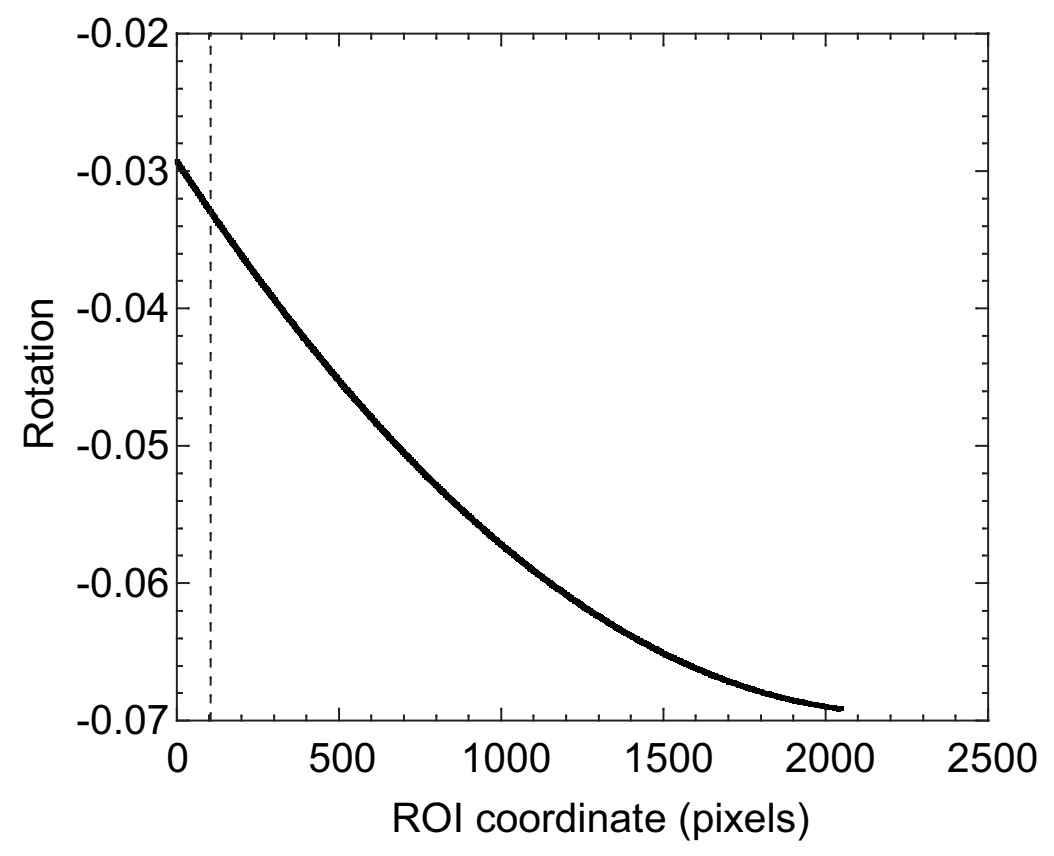

(a)



(b)

Fig. 8. Rotation field for the last load level (a). A rotation discontinuity is observed on the left end of the ROI. The location of the weld is depicted by the dashed line. Rotation field for the last load level when different numbers of elements are used (b). When at least four elements are used, the measurement is regularized on $75 \%$ of the beam length and the base rotation is prescribed on the left part of the ROI. 


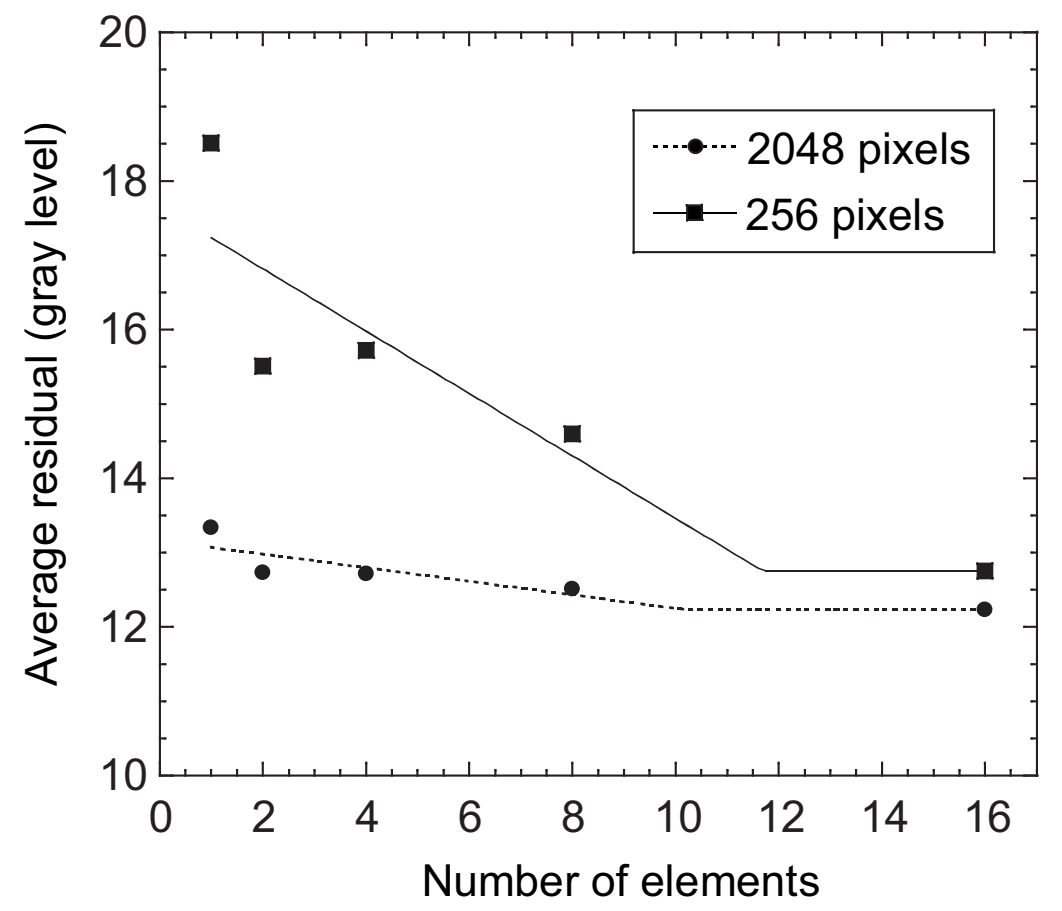

Fig. 9. Average residuals (in gray levels) as a function of number of beam elements. Two zones are considered, the first 256 pixels and the whole 2048-pixel ROI to evaluate the average value. The curves depict trends in terms of change of the correlation residual with the chosen discretization. 


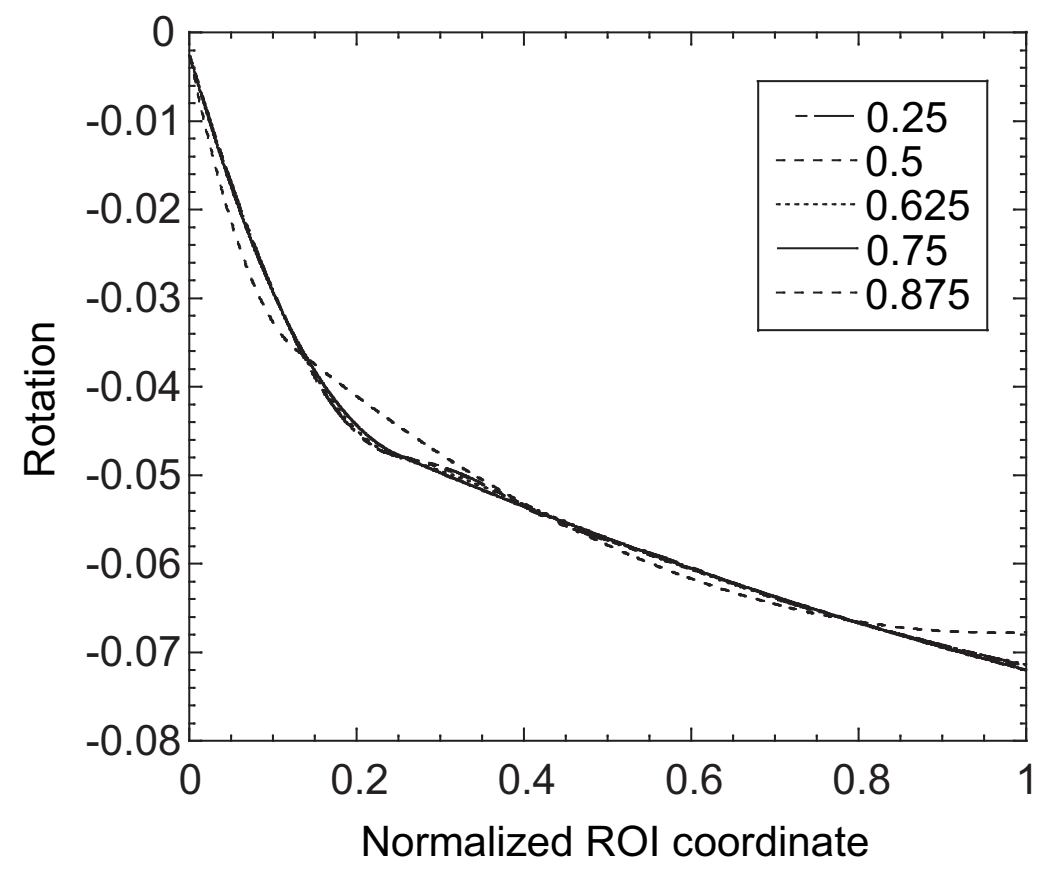

(a)

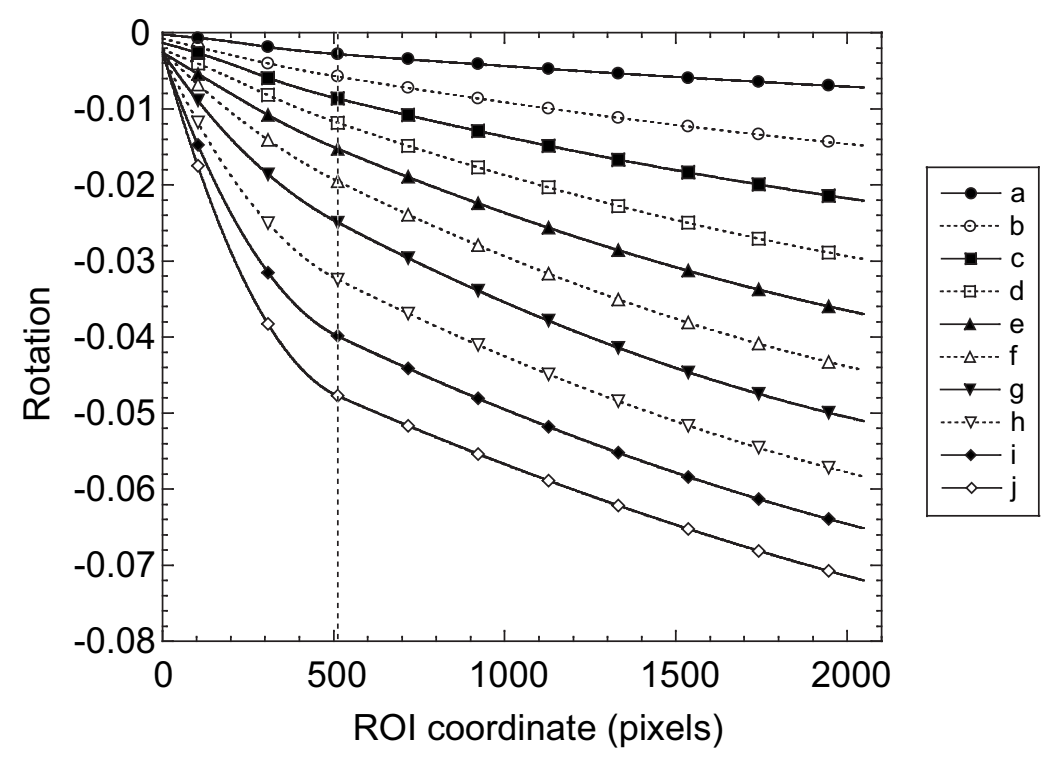

(b)

Fig. 10. Rotation field for the last load level when different percentages of the right part of the ROI length are regularized (a). Rotation fields for the ten analyzed load levels (b). The dashed line depicts the location where the regularization starts. 


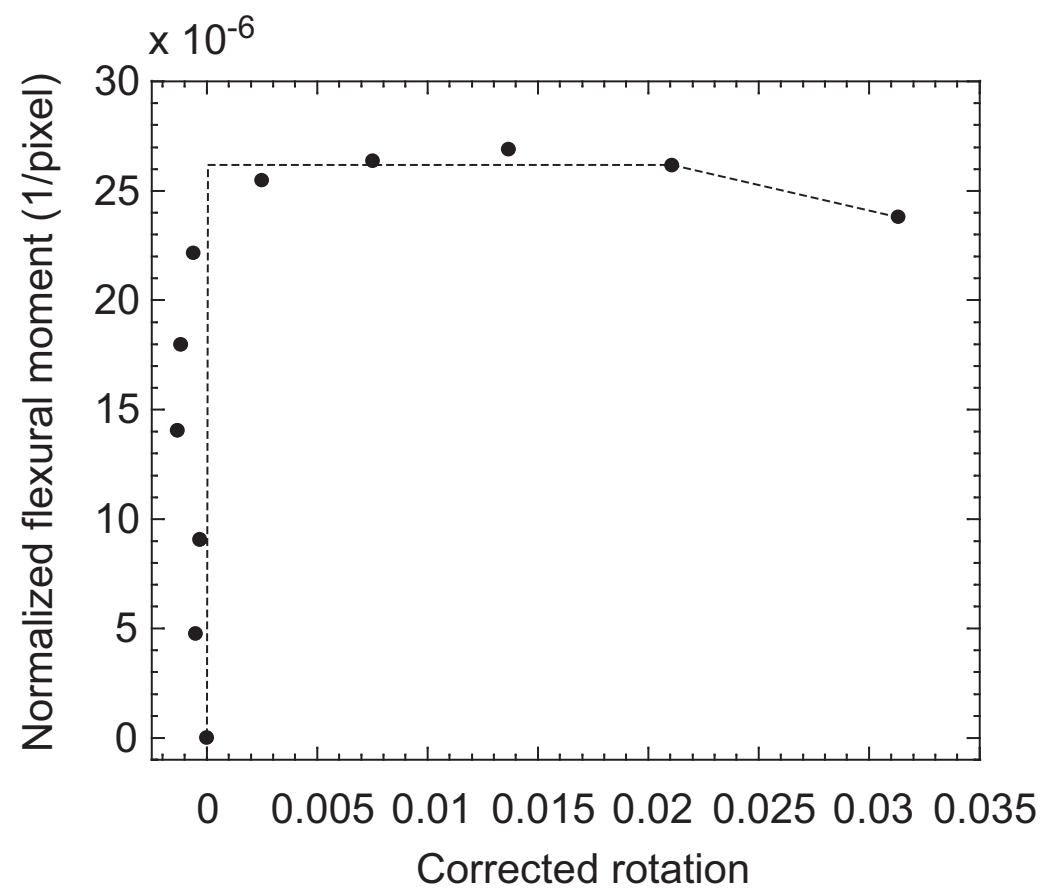

Fig. 11. Normalized flexural moment $M / E I_{0}$ vs. corrected section rotation $\Theta$ for the analyzed experiment. The dashed line shows the identification result with the lumped model [Equations (14-16)].

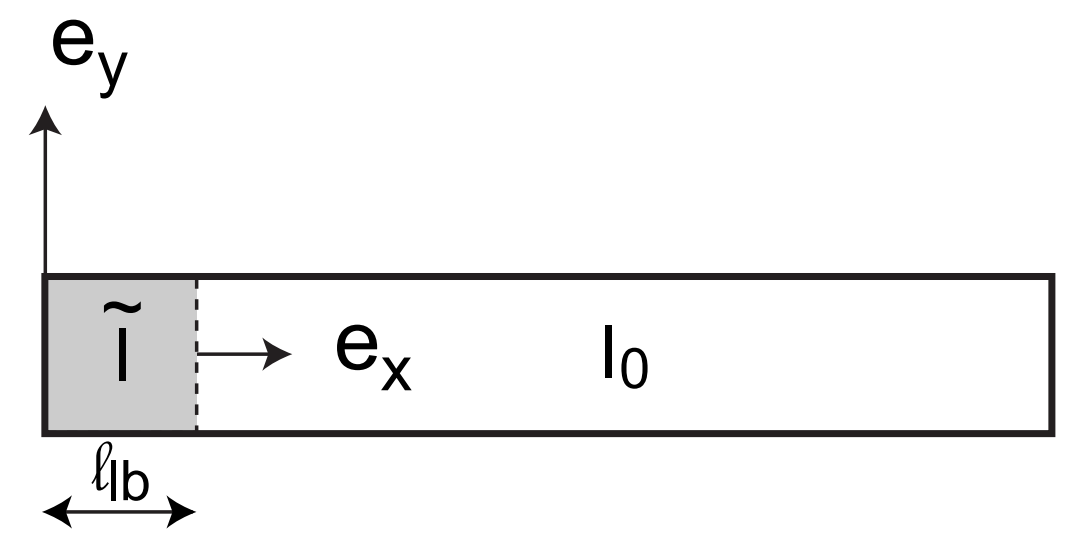

Fig. 12. Beam with a degraded inertia $\tilde{I}$ over a length $\ell_{l b}$ with respect to a reference inertia $I_{0}$. 


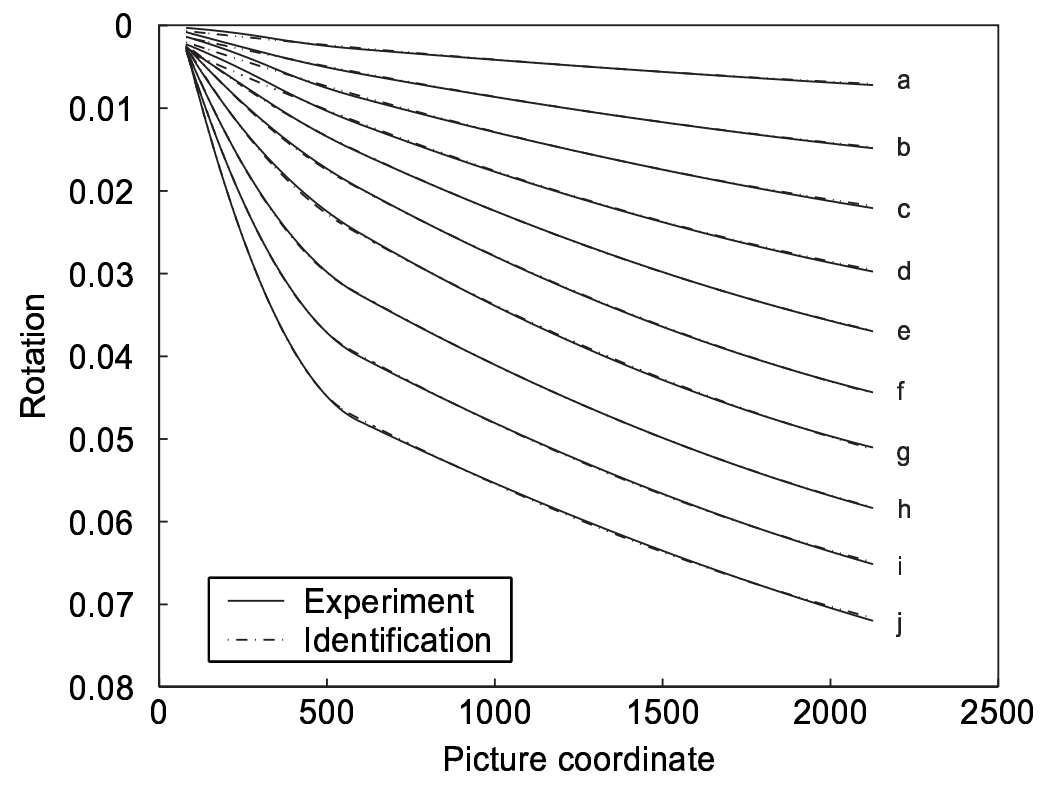

(a)

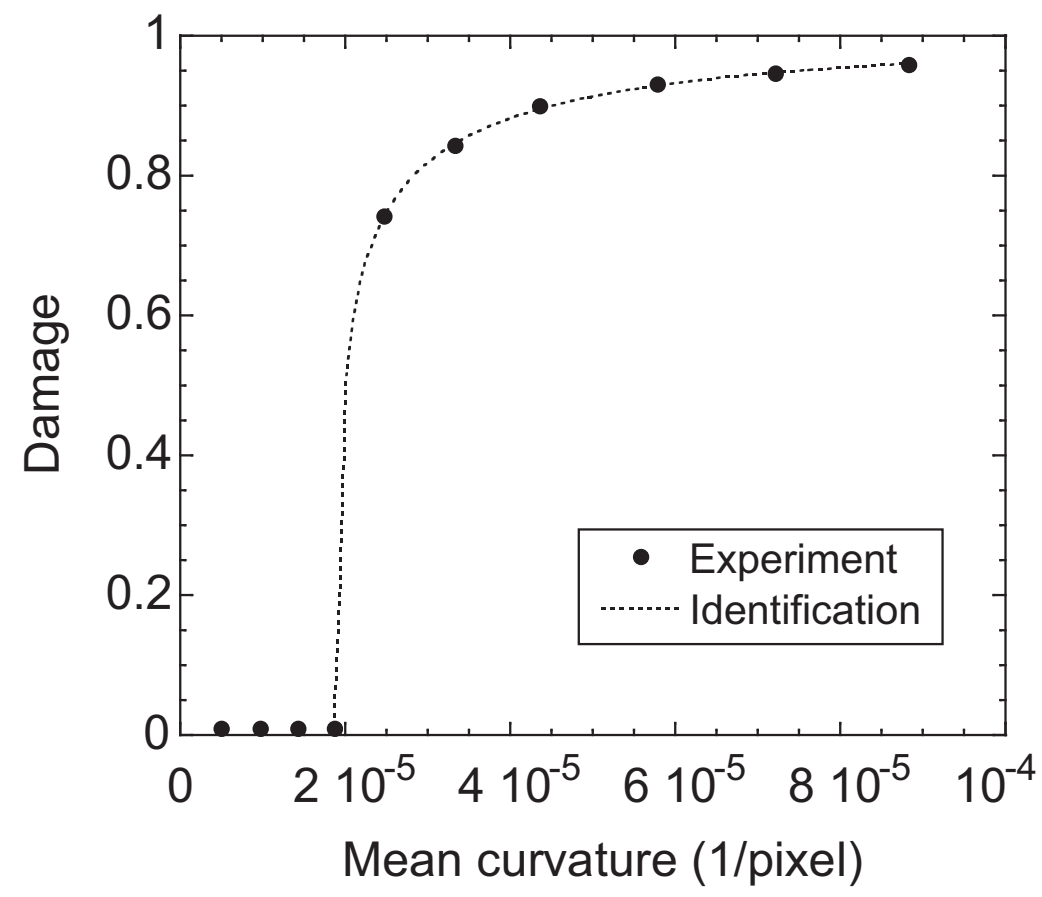

(b)

Fig. 13. Rotation fields for the ten analyzed load levels (b). Comparison between experimental and predicted rotations when a constant inertia degradation is assumed in the left part of the beam (b). Damage parameter $\tilde{D}$ vs. average curvature $\tilde{\kappa}$. The dashed line corresponds to the best fit with the damage law (21). 